\title{
A New Dynamic Self-Organizing Method for Mobile Robot Environment Mapping
}

\author{
Xiaogang Ruan, Yuanyuan Gao, Hongjun Song, Jing Chen \\ Institute of Artificial Intelligence and Robots, Beijing University of Technology, Beijing, China. \\ Email: gyyshj@yahoo.cn
}

Received June 19 ${ }^{\text {th }}$, 2011; revised July 19 ${ }^{\text {th }}$, 2011; accepted August $1^{\text {st }}, 2011$.

\begin{abstract}
To solve the mapping problem for the mobile robots in the unknown environment, a dynamic growing self-organizing map with growing-threshold tuning automatically algorithm (DGSOMGT) based on Self-organizing Map is proposed. It introduces a value of spread factor to describe the changing process of the growing threshold dynamically. The method realizes the network structure growing by training through mobile robot movement constantly in the unknown environment. The proposed algorithm is based on self-organizing map and can adjust the growing-threshold value by the number of network neurons increasing. It avoids tuning the parameters repeatedly by human. The experimental results show that the proposed method detects the complex environment quickly, effectively and correctly. The robot can realize environment mapping automatically. Compared with the other methods the proposed mapping strategy has better topological properties and time property.
\end{abstract}

Keywords: Mobile Robot, Environment Mapping, Growing-Threshold Tuning, Self-Organizing

\section{Introduction}

The ultimate goal of mobile robotics research is to endow the robots with high autonomous ability, of which navigation in an unknown environment is achieved by using on-line sensory information. First a correct environment model is usually needed and the robot can update the model by using sensory information. It is a key problem that the robot is able to map the environment automatically. A significant amount of research effort has been devoted to this area in the past decades such as probability grid [1], geometry algorithm [2], topology information [3] and the 3D information methods [4]. The deficiency of geometry algorithm is that feature extraction is very difficult, especially in the complex environment. On the other hand, the topological method has the higher efficiency and smaller memory space. It is suitable for the large-scale work space. By this approach, main concern lays in finding the most effective way to map the environment while simultaneously localizing the robot's position relative to the map. If just the accurate map were acquired, then the shortest path would be easily obtainable from the occupancy grid map [5]. However, to acquire such map in an unknown environment is not easy. The robot moves in the unfamiliar and non-stationary environment and doesn't know any prior knowledge.
Network growth is an important feature to adapt to non-stationary environments. Many conventional clustering algorithms such as k-means demand that a user predetermine how many clusters are to be generated. For a topology learning problem, a user of many conventional methods like Kohonen Feature Map [6] must decide the number of nodes in advance.

Nehmzow and Smithers have used Kohonen's Selforganizing Maps (SOMs) to endow their robots with map building abilities [7]. Their approach to mapping is based on the correlations that exist between the sensor data representing a fixed environment and the motor responses of the robot. Najand et al. have been working along the same line using TPM (Topology Preserving Mapping) networks. They studied problems associated with parameters which affect the learning process [8]. V. Morellas, et al. proposed an approach which puts emphasizes the dynamic interaction between the mobile robot and the environment and attempts to bridge the gap between Artificial Intelligence (AI) techniques and behavior-based methodologies [9]. A major problem with this solution is that the set of vertices corresponding to the objects of the environment must be known a priori, which in gener- al, is a difficult and computationally time consuming task.

Incremental learning addresses the ability of repeatedly training a network using new data without destroy- 
ing the old prototype patterns. Incremental learning is useful in many applications. For example, if we intend to bridge the gap between the learning capabilities of humans and machines, we must consider which circumstances allow a sequential acquisition of knowledge. The fundamental issue for incremental learning is how a learning system can adapt to new information without corrupting or forgetting previously learned information [10].

In recent years, many scholars have also applied some methods of incremental learning to the robot environment map. Oishi, T. et al. designs the architecture of a self creating and organizing neural network for workspace recognition and navigation of an autonomous mobile robot. Methods of path planning and navigation based on a topological map created by learning are also proposed. The proposed architecture was tested by simulation of an autonomous mobile robot with eight sonar sensors, and it was demonstrated that the architecture is useful for the purpose [11]. Gyu-jong Choi used the topological form to build the environment map [12]. Y. Zhuang et al. had maked use of the geometry-topoloty hybrid approach [13]. A. Kawewong et al. used an approach based on the associative memory using Self-Organizing Incremental Neural Networks (SOINN) because it is suitable to noisy environment and is easily implemented [14]. Ruan et al. presented an algorithm of Dynamic Growing Self-organizing (DGSOM) using in building environment [15]. It is simple and effective but the static value of Growing Threshold (GT) is used which can not realize mapping quickly.

However, it is not easy to define appropriate search and memory space. To solve this problem, a dynamic growing self-organizing map with growing-threshold autonomic tuning algorithm (DGSOMGT) based on Selforganizing Map is proposed and it is used in environment mapping. The spread factor (SF) is introduced to describe the degree of TPM. The value of GT changes with the value of SF. If SF is smaller, GT is bigger and it can realize the lower clustering. Or else, it can realize the higher clustering. The proposed algorithm is based on selforganizing map and can adjust the growing-threshold value by the number of network neurons increasing. It avoids tuning the parameters repeatedly by human. The experimental results show that the proposed method detects the complex environment effectively and correctly and the robot can realize environment mapping automatically. Compared with the SOM method the proposed mapping strategy has better topological properties and time.

\section{SOM Algorithm Introduction}

A new idea is presented by the appearance of Self-organizing Map. The Kohonen feature map [6] allows projection onto non-linear, discretely sampled subspaces of a dimensionality. But it requires predetermination of the network structure and network size. The combination of "competitive Hebbian learning" (CHL) and "neural gas" (NG) [16] also requires a prior decision about the network size. Therefore, the Growing Cell Structure [17], SelfCreating and Organizing Neural Networks [18] and Growing Self-Organizing Map [19] were presented to solve the problem in the information processing field such as image processing, pattern recognition and data compression. Shen and Hasegawa proposed an incremental learning method called the self-organizing incremental neural network (SOINN) [20] to realize the unsupervised incremental learning task. In fact, SOINN is useful to process online non-stationary data, report a suitable number of classes, and represent the topological structure of input probability density. An enhanced self-organizing incremental neural network (ESOINN) [10] is proposed to accomplish online unsupervised learning tasks. It improves the self-organizing incremental neural network (SOINN).

The above methods are all based on the algorithm of SOM. Here we introduce the process of SOM firstly.

An n-rank SOM of n-dimensional input space can be determined exclusively by the feed forward synaptic connection. It has two layers of the input layer and competitive layers. Competition, cooperation and synaptic adaptation constitute are the three basic aspects of SOM operational mechanism.

1) SOM Competition mechanism

SOM competition mechanism is:

$$
o_{i(x)}(x)=\min \left\|x-w_{j}^{T}\right\|
$$

where $x$ is the input of stimulus. $v_{i(x)}$ is the connection weight of the neuron number of $\mathrm{j} . o_{i(x)}(x)$ is the output of winning unit. Neuron $v_{i(x)}$ will become the winning unit which is stimulated by $x$. The winning SOM neuron $v_{i(x)}$ is the excited centre of SOM sensory field which is stimulate by $x$.

2) SOM Cooperation Mechanism

Winning SOM neurons sets up a topological neighborhood with taking itself as the center. Like in biological nervous system, the winning neuron stimulates neighborhood neurons to excite together with a distribution similar to Mexican Hat Function. Neighborhood function can be a square wave function or a Gaussian function. The most commonly use are Gaussian functions:

$$
\omega_{i(x) j}=\exp \left\{-\frac{d_{i(x) j}^{2}}{2 \sigma^{2}}\right\}(j=1,2, \cdots, N)
$$

where $\sigma$ is the effective radius of Gaussian neighborhood, $\alpha$ is lateral distance, that is, Euclidean distance between neuron $v_{j}$ and the winning neuron.

3) SOM Synaptic Adaptation Mechanism 
SOM synaptic adaptation mechanism is the process of self-organization that modifies or adjusts the synaptic contact efficiency of SOM neurons

SOM synaptic adaptation law as follows:

$$
\left\{\begin{array}{l}
\Delta w_{j}(t)=\alpha(t) \omega_{i(x) j}(t)\left(x-w_{j}(t)\right) \\
w_{j}(t+1)=w_{j}(t)+\Delta w_{j}(t)(j=1,2, \cdots, N)
\end{array}\right.
$$

In addition, the GCS, SCONN and GSOM derived by SOM which have the different network structure and the new node generation condition but train the network through the same method of the best match and neighborhood weight value adaptation. The adjacency relation of SCONN and GCS is relatively complex. GSOM cannot generate a new node in the suitable location. SOINN and ESOINN are not suitable for the complex problems. DGSOM has a static value of GT and it is real time. DGSOMGT by this paper presented allows dynamic generation in the suitable place and but also avoids the complex of the adjacency relations.

\section{The Algorithm of DGSOMGT}

In DGSOMGT algorithm, the Euclidean distance between input samples and the connection strength of winning neuron is the standard to determine whether increase neurons:

$$
d_{i(x)}=\left\|x-w_{i(x)}^{T}\right\|
$$

When $d_{i(x)}$ is more than a given growth threshold GT, the algorithm considers the current SOM scale is not sufficient to describe the characteristic of samples, then adds a SOM neuron, and sets its initial feed forward connection weights $w_{q}=x^{T}$, and then looks for its neighborhood neurons, sets up neighborhood connections.

The algorithm of DGSOMGT summarized as the following:

Step 1: Initialization parameters: Set the synergistic parameters $\left(\sigma_{0} \kappa_{\sigma}\right)$, self-adaptive parameters $\left(\alpha_{0} \kappa_{\alpha}\right)$, the initial SOM mumble $n_{\text {init }}$, Spread Factor SF, the input sample number N_sample, connection weight

$\left\{w_{j}(0)\right\}_{j=1}^{N}=0$, the training time $t=0$.

Step 2: Training.

Step 2.1: Generating samples $x(t) \in D_{\mathrm{SOM}}^{(n)}$ randomly.

Step 2.2: Competition. According to the formula (1) to determine the winning unit $v_{i(x)}$.

Step 2.3: Growing Judgment. If $o_{i(x)}(x)>\mathrm{GT}$, add a new unit and go to Step 2.4. if not, $n=n+1$, go to Step 2.5. Here, add the spread factor SF to calculate $\mathrm{GT}=$ $f(\mathrm{SF}), 0<\mathrm{SF}<1,0<f(\mathrm{SF})<1$. If $\mathrm{SF}$ is smaller, $\mathrm{GT}$ is bigger and it can realize the lower clustering. Or else, it can realize the higher clustering.

$$
\mathrm{GT}=f(\mathrm{SF})=\frac{(1-\mathrm{SF})^{2}}{1+n_{\text {init }} / n(t)}
$$

where $n(t)$ is the number of the net. When $n(0)=n_{\text {init }}$, GT for half processing can improve its activation level. Through the growing of $n(t)$, the effect of $1+n_{\text {init }} / n(t)$ reduce. So the user designs the SF in indicial process and then gets the GT.

Step 2.4: Add a new neuron. $N \_s o m=N \_s o m ~+1$, the connection weights $w_{\mathrm{N} \_ \text {som }}=x(t)^{T}$, determining its neighborhood neurons by GSOM_NBN algorithm.

Step 2.5: Cooperation. According to (2) calculating the value $\omega_{i(x) j}$ of $v_{i(x)} \rightarrow v_{j}$.

Step 2.6: Synaptic adaptation. According to SOM Synaptic Adaptation Mechanism, Calculating feed forward connection strength $w_{j}(t+1)(j \in\{1,2,3 \cdots, N\})$.

Step 2.7: Stop. If $n=$ N_sample, go to Step 3; if not. T $=t+1$ and then go to Step 2.1

Step 3: Smooth. Reduce the synergistic neighborhood radius $\sigma$ and the learning rate $\alpha$ and further tune $w_{j}(t+1)(j \in\{1,2,3 \cdots, N\})$. Training Step 2 repetitively until $\mathrm{t}=\mathrm{T}$. This step may also sets the different SF for the interest regions to realize hierarchical clustering.

In the Step 2.4, determining the neighborhood neurons based on GSOM_NBN algorithm [11]. We can see the structure map of GSOM_NBN in Figure 1. The algorithm is described as follows:

Step 1: Calculating the distance $d(i)$ between the new neuron and all the other neurons.

Step 2: Searching all the neurons in $N 1$ that $d(i)<n 1 * \mathrm{GT}$ and all neurons in $N 2$ that $d(i)<n 2 * \mathrm{GT} \quad(n 1<n 2)$.

Step 3: Judging whether the connection relations established between the new neuron and neurons in N1 have intersected the connection relations established between any two neurons in $N 2$. Neurons which connect with new neuron without intersecting any other connections are in $N 3$.

Step 4: Setting up connections between the new neuron and all neurons in $N 3$ which is identified as the neighborhood neurons.

In the process for searching the neighborhood neurons, it is required to set the value of $n 1$ and $n 2$ in advance, that is, to set the range for searching neighborhood neurons. The topological structure of the topological map is

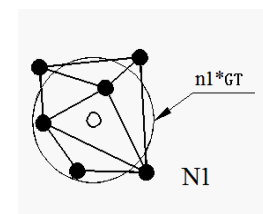

(a)

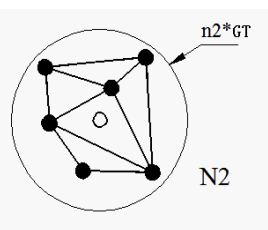

(b)

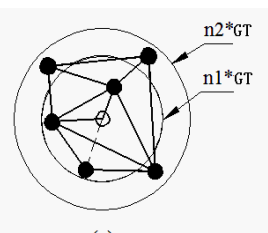

(c)
Figure 1. The structure map of GSOM_NBN. 
different with different $n 1$ and $n 2$. If $n 2$ is too large, it will reduce the efficiency of algorithm. If $n 2$ is too small, it will form unnecessary cross connections. If $n 1$ is too large, it will also reduce the efficiency of algorithm. If $\mathrm{n} 1$ is too small, it will result in some neighborhood neurons can not be found. Generally set $2<n 1<n 2<3^{*} n 1$.

The features of DGSOMGT are:

- The initial number of network neurons can be set randomly.

- To describe the clustering degree by SF and the GT value is related with the number of neurons.

- Support multidimensional sample inputting.

\section{Environment Mapping Based on DGSOMGT}

\subsection{Mobile Robot Model and Coordinate Systems}

We use a differential drive cylindrical mobile robot model with radius of $\mathrm{R}=20 \mathrm{~cm}$. The robot is equipped with 6 ultrasonic sensors evenly distributed in the front as depicted in Figure 2(a). Each sensor, $S_{i}$ for $i=1, \cdots, 6$, covers and angular view of $30^{\circ}$ and gives the distance to the obstacle $L_{i}$ in its field of view.

Two coordinate systems is used: the world coordinate system XOY and the mobile robot coordinate system xoy where $\mathrm{o}$ is the center of the robot and the $\mathrm{x}$ axis goes through $S_{3}$ as depicted in Figure 2(b). The robot actions are the change of the heading angle $\phi$ and the

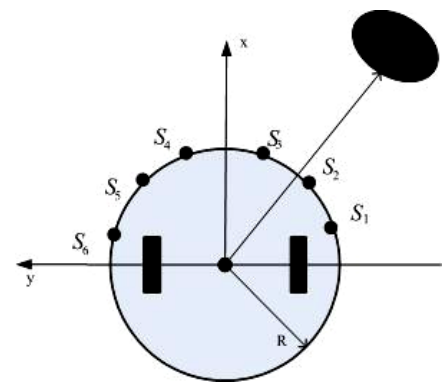

(a)

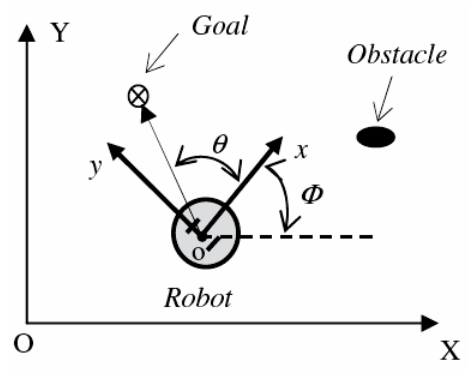

(b)

Figure 2. The mobile robot and the system coordinate. XOY is the world coordinate and XOY is the robot coordinate. Goal and Obstacle are marked respectively. linear velocity $v$ of the robot. For a goal seeking behavior, the robot knows the position of its goal and $\theta$ defined as the angle between the orientation axis and the line connecting the centre of the robot to the goal.

\subsection{Environment Mapping Process}

For the environment mapping, we assume that the effecttive range of the ultrasonic sensors is $10 \mathrm{~cm} \mathrm{-} 210 \mathrm{~cm}$ and the velocity of mobile robot is $20 \mathrm{~cm} / \mathrm{s}$. A time step of $1 \mathrm{~s}$ was used and the minimum and maximum steering in a time step are $-30^{\circ}$ and $30^{\circ}$.The robot safe distance to obstacle is set to be $20 \mathrm{~cm}$. The initial values of the other parameters used for the simulation are tabulated in the Tables 1 and 2.

The size of robot moving environment is $5.5 \mathrm{~m} \times 4 \mathrm{~m}$. The range of $\mathrm{x}$ coordinate is $(2.5 \mathrm{~m}, 8 \mathrm{~m})$ and $\mathrm{y}$ coordinate is $(3.3 \mathrm{~m}, 7.3 \mathrm{~m})$. The robot can moving in the environment which is shown in Figure 3.

The robot moves in the unknown environment without collision firstly. The experiment is done using DGSOMGT with SF $=0.4$. The blue “.” represents the coordinate position of robot moving and the red “-” represents topological structure in Figure 4.

We can see the mapping learning process from the

Table 1. Initial simulation parameters for SOM.

Ordering Phase learning rate $\alpha_{1} \quad$ Tuning Phase learning rate $\alpha$

\begin{tabular}{ll}
\hline 0.9 & 0.02 \\
\hline
\end{tabular}

Table 2. Initial simulation parameters for DGSOMGT

\begin{tabular}{cccccc}
\hline$\sigma_{0}$ & $\alpha_{0}$ & $k_{\sigma}$ & $k_{\alpha}$ & $n_{\text {init }}$ & $n 1$ \\
\hline 0.9 & 0.9 & 0.0001 & 0.001 & 1 & 2.2 \\
$S F(1)$ & $S F(2)$ & $\sigma_{0}$ & $\alpha_{0}$ & $n 2$ & N_sample \\
0.4 & 0.2 & 0.2 & 0.02 & 6 & 2000 \\
\hline
\end{tabular}

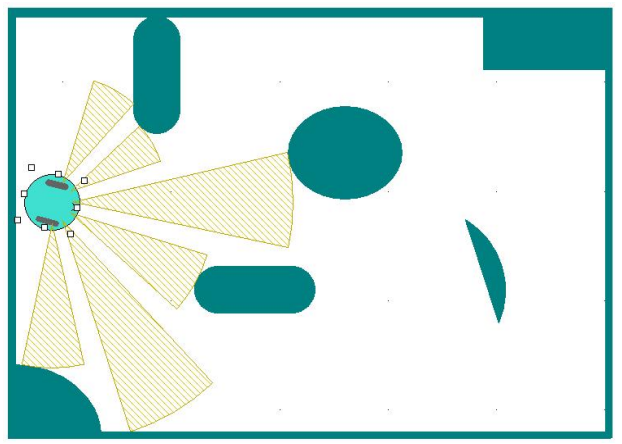

Figure 3. Environment mapping for simulation. The green irregular shape object represent obstacles. 
Figure 4(a) to (d) when the robot moving in the environment. The numbers of neuron in (a) (b) (c) are 19, 39, 59 , and the last simulation number is 76 . It just used 76 neurons to descript the 2000 location information. So the method is sufficiently, correctly, and completely for environment mapping.

The dynamic tuning of GT can be through SF in DGSOMGT algorithm. We just research on the two conditions dynamic GT with SF and static GT without SF.

The initial parameters of experiment are in Table $\mathbf{1}$ and Table 2. Figure 5(a) is the result using SOM and $\mathbf{5 ( b )}$ is the algorithm of DGSOM [11] with GT $=0.35$. The algorithm of SOM requires predetermination of the network structure and network size. Set N_som $=9 * 9=81$ of SOM and N_som = 76 at last using DGSOMGT. In (a), SOM method is used which can't express the environment information correctly. And we can see from (b), it doesn't using SF and the value of GT is static. Set GT = 0.35 , it uses 76 neurons to express the circumstances map

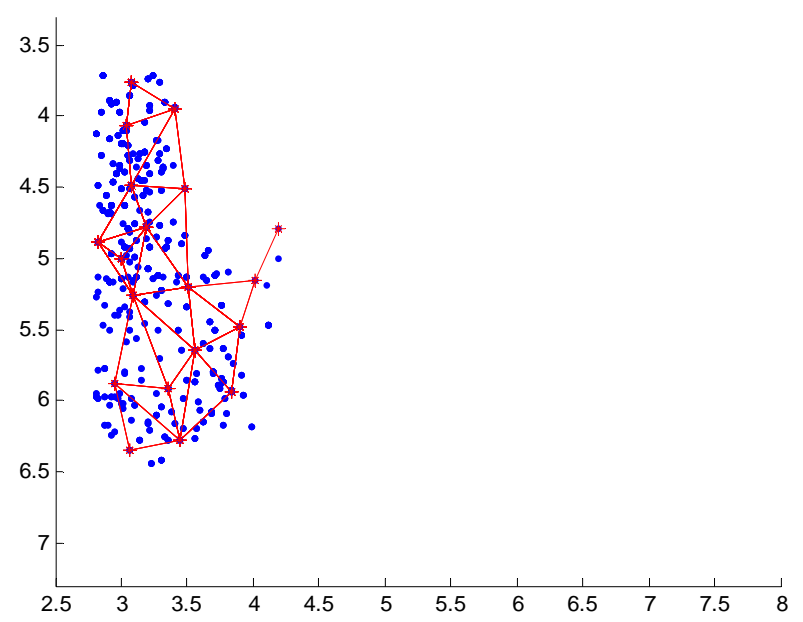

(a)

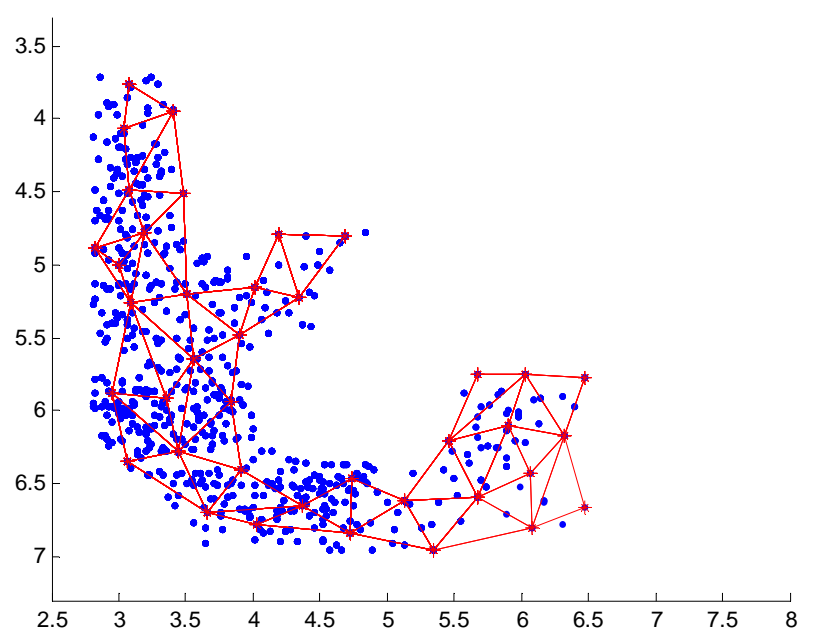

(b)

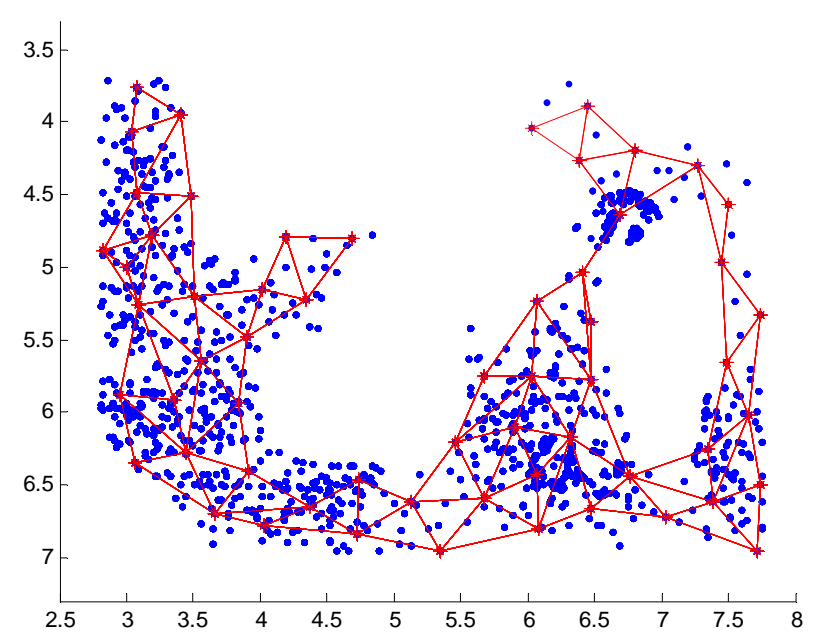

(c)

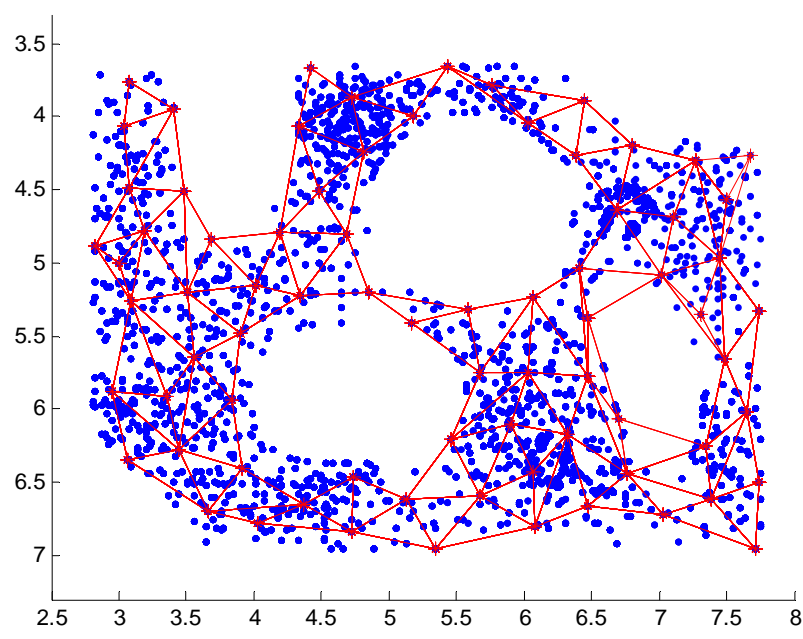

(d)

Figure 4. Environment mapping process of the number of neuron 19, 39, 59 and 76 respectively using DGSOMGT. (a) (N_som = 19; (b) N_som = 39; (c) N_som = 59; (d) N_som = 76 .

accurately but spending a long time to mapping which is shown in Figures 6 (c) and (d) are used our method of DGSOMGT with $\mathrm{SF}=0.4$ and $\mathrm{SF}=0.2$. If $\mathrm{SF}$ is smaller, GT is bigger and it can realize the lower clustering. Or else, it can realize the higher clustering. The value of GT can be achieved by equation (5) in section 3 .

From Figures 5(b) and (c), they have the similar Topology Preserving Mapping when using the methods of DGSOM and DGSOMGT with 76 neurons. But the mapping generation times are very different. The algorithm of DGSOM spends about 2250s but DGSOMGT uses only about 1250s which can be seen from Figure 6. The blue dotted line represents DGSOM with simulation in Figure 5(b) and the block line represents DGSOMGT with simulation in Figure 5(c). Two networks have the same neurons but the time using is littler by DGSOMGT. 
It is because that the value of GT is dynamic and it has a smaller value in the early mapping when using the algorithm of DGSOMGT. The Topology Preserving Mapping can grow more quickly than using the algorithm of DGSOM.

In Figures 5(c) and (d), the method of DGSOMGT is used with different SF. From Section 3 we know that the value of SF is bigger and the Topology Preserving Mapping is more detailed, but the time spending is growing accordingly.

In the real time Topology Preserving Mapping, time consumption is a very important performance to identify the algorithm practicality.

Figure 6 shows the three growing curves of neuron number with the time consumption using the algorithm of DGSOM and DGSOMGT with $\mathrm{SF}=0.4$ and $\mathrm{SF}=0.2$. We can see that two networks have the same neurons but the time consumption is different using DGSOM with $\mathrm{GT}=0.35$ and DGSOMGT with $\mathrm{SF}=0.4$ which the value of GT is changing from 0.18 to 0.35 . The time consumption of DGSOM is longest which is about 2250s,

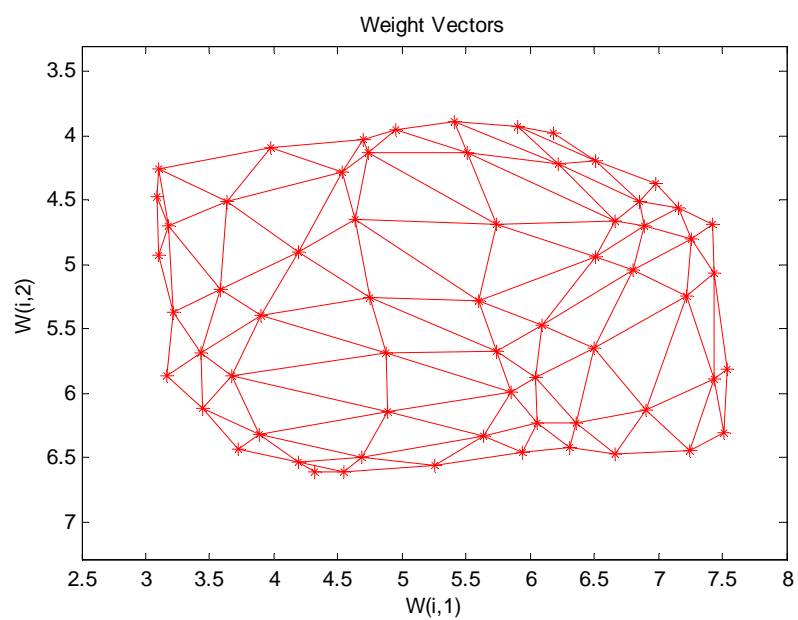

(a)

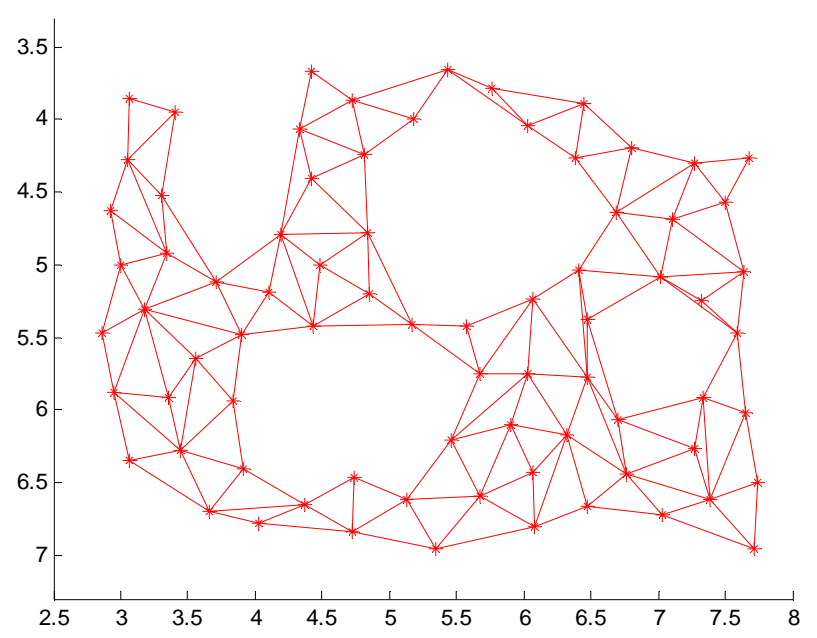

(b)

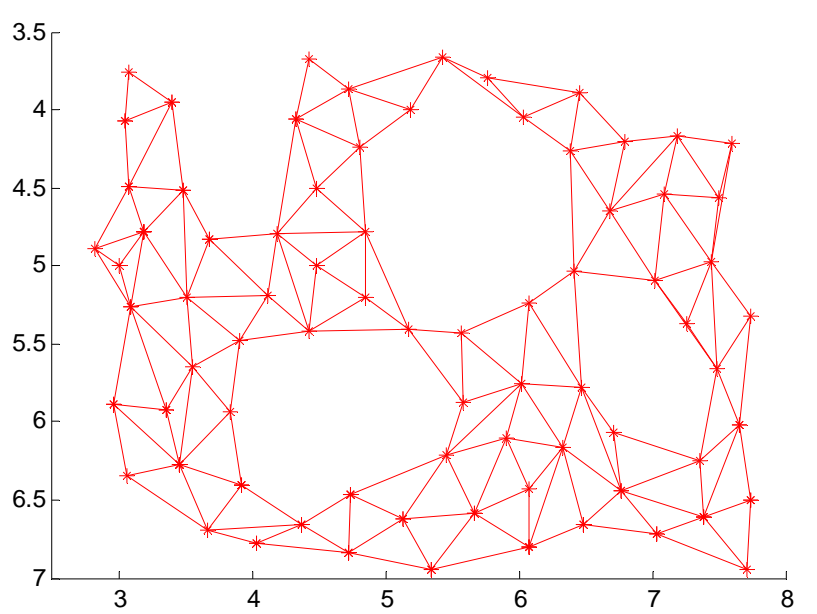

(c)

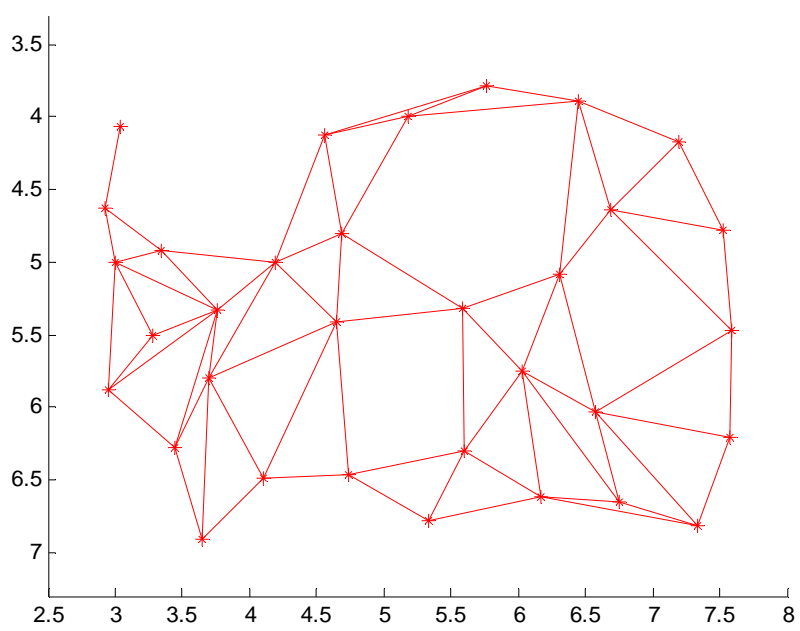

(d)

Figure 5. The experiments of environment mapping are realized by SOM, DGSOM and DGSOMGT respectively. The $\mathrm{X}$-axis is the horizontal ordinate of $2 \mathrm{D}$ environment, and the $\mathrm{Y}$-axis is the vertical coordinates. The simulation experiments using DGSOM with static GT $=0.25$, and DGSOMGT with $\mathrm{SF}=0.4$ and with $\mathrm{SF}=0.2$.

and DGSOMGT with SF $=0.4$ which is about 1250 s. The time is reduced by about 1000s when introducing SF. The red dotted line represents the result of DGSOMGT with $\mathrm{SF}=0.2$. It has the shortest time but not correct description when the value GT changes from 0.33 to 0.58 . The values of GT are too big for mapping when SF is equal to 0.2. At last, we find a more suited value of SF to building the environment mapping which $\mathrm{SF}$ is equal to 0.4 consideration of TPM and time consumption.

Figure 7 shows the change value of GT with the robot moving in the environment when SF is equal to 0.4 and 0.2 . When the value of SF is bigger, the GT is smaller and it can describe the environment accurately but time consuming. If the value of SF is too smaller, the Topol- 
ogy Preserving Mapping is not correct for the real environment which can be seen form Figure 5(d) with SF = 0.2.

State-Graph Search is the most important solve method in artificial intelligence symbols computing science. The SOM map can be seemed as the state graph. To solve the goal seeking problem and find the optimal path without obstacle collision, here we use A* algorithm. A* algorithm is an optimization algorithm for heuristic search, by it we can assure to get the optimal solutions in every step of the search. And the search based on it may be looked as a process to search and find the goal node from the start source node in the state-space graph. It chooses and maintains the nodes by an open table and a closed table.

In Figure 8, we set the Start location (3.5 m, $5.2 \mathrm{~m})$ and the Goal location $(7.5 \mathrm{~m}, 6 \mathrm{~m})$. At last the robot can find a path from start to goal, which the black line was shown.

In this paper, we have proposed an algorithm of

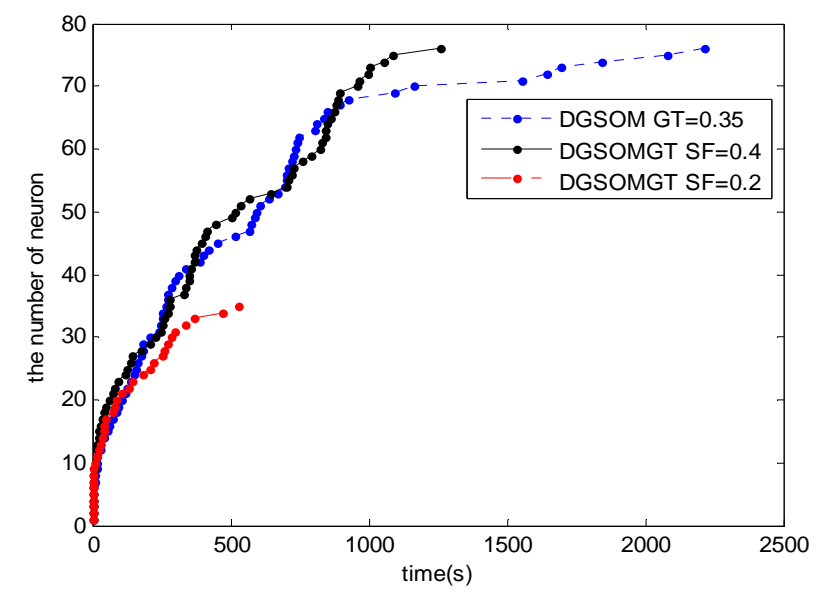

Figure 6. Three growing curves of neuron number with the time consumption using DGSOM with GT $=0.35$, DGSOMGT with $\mathrm{SF}=0.4$ and $\mathrm{SF}=0.2$.

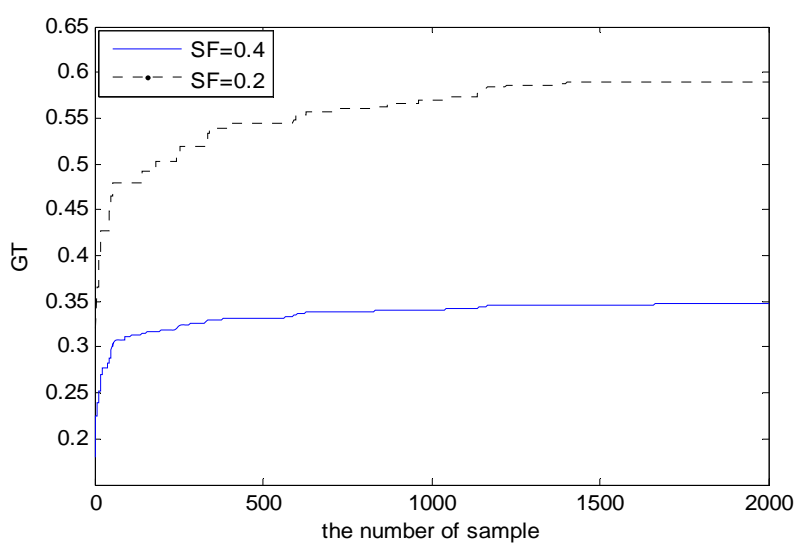

Figure 7. The value of GT curve by DGSOMGT with SF = 0.4 and $\mathrm{SF}=0.2$.

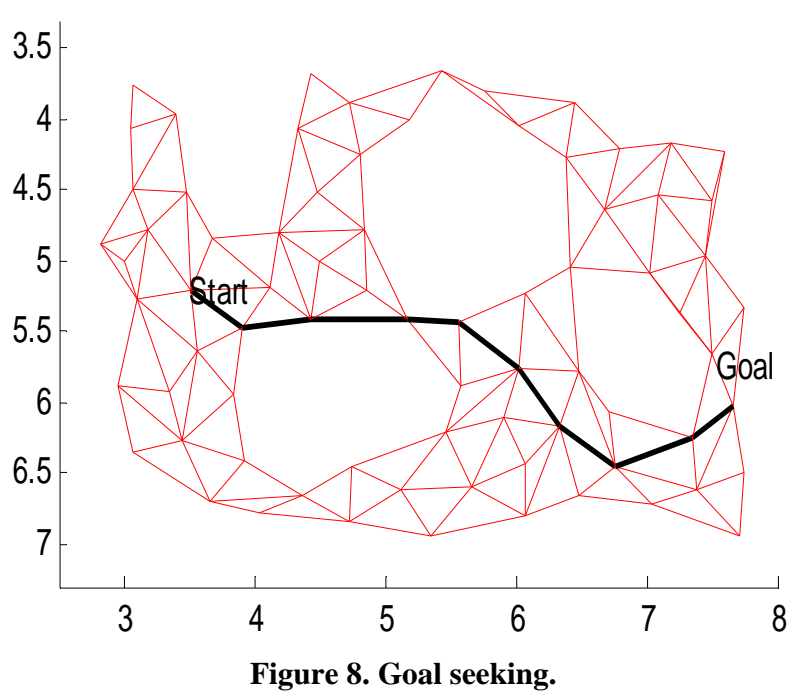

DGSOMGT and a solution to the environment mapping problem in navigation for mobile robots in unknown environments based on it. The proposed method is based on self-organizing map and can adjust the growing-threshold value by the number of network neurons increasing. It avoids tuning the parameters repeatedly by human. This method endows the robot with capabilities of obstacle avoidance and goal seeding without based on the environment position map. The merits of the proposed method are its little time loss and parameters tuning automatically. The efficiency of the method is demonstrated through simulation results.

\section{Acknowledgments}

We acknowledge support from National Natural Science Foundation of China (No. 61075110), China's 863 Program (No. 2007AA04Z226), Key Project (KM2008 10005016) of S\&T Plan of Beijing Municipal Commission of Education, and Beijing Natural Science Foundation (No. 4102011).

\section{REFERENCES}

[1] H. P. Moravec and A. Elfes, "High Resolution Maps from Wide Angle Sonar,” IEEE International Conference on Robotics and Automation, St. Louis, 25-28 March 1985, pp. 116-121.

[2] B. Kuipers and Y. T. Byun, “A Robot Exploration and Mapping Strategy Based on a Semantic Hierarchy of Spatial Representations," Journal of Robotics and Autonomous Systems, Vol. 8, No. 1-2, 1999, pp. 47-63. doi:10.1016/0921-8890(91)90014-C

[3] R. Chatila and J. P. Laumond, "Position Referencing and Consistent World Modeling for Mobile Robots," IEEE International Conference on Robotics and Automation, St. Louis, 25-28 March 1985, pp. 138-145.

[4] D. Avots, E. Lin, R. Thibaux, et al., "A Probabilistic 
Technique for Simultaneous Localization and Door State Estimation with Mobile Robots in Dynamic Environments," Proceedings of IEEE/RSJ International conference on Intelligent Robots and System, Lausanne, 30 September-5 October 2002, pp. 521-526.

[5] B. Kuipers, J. Modayil, P. Beeson, M. Macmahon and F. Savelli, "Local Metrical and Global Topological Maps in the Hybrid Spatial Semantic Hierarchy," Proceedings of International conference on Robotics and Automation, New Orleans, 26 April-1 May 2004, pp. 4845-4851.

[6] T. Kohonen, "Self-Organized Formation of Topologically Correct Feature Maps,” Biological Cybernetics, Vol. 43, No. 1, 1982, pp. 59-69. doi:10.1007/BF00337288

[7] U. Nehmzow and T. Smithers, "Mapbuilding Using SelfOrganizing Networks in Really Useful Robots," From Animals to Animats: Proceedings of the First International Conference on Simulation of Adaptive Behavior, Paris, 24-28 September 1991, pp. 152-159.

[8] S. Najand, Z. Lo and B. Bavarian, "Application of SelfOrganizing Neural Networks for Mobile Robot Environment Learning," Neural Network in Robotics, Kluwer Academic Publishers, Vol. 202, No. 1, 1993, pp. 85-96.

[9] V. Morellas, J. Minners and M. Donath, "Implementation of Real Time Spatial Mapping in Robotic Systems through Self-Organizing Neural Networks," Proceedings of 1995 IEEE/RSJ International Conference on Intelligent Robots and Systems. Human Robot Interaction and Cooperative Robots, Vol. 1, Pittsburgh, 5-9 August 1995, pp. 277-284.

[10] F. Shen, O. Hasegawa and H. Osamu, "An Enhanced SelfOrganizing Incremental Neural Network for Online Unsupervised Learning," Neural Networks, Vol. 20, No. 8, 2007, pp. 893-903. doi:10.1016/j.neunet.2007.07.008

[11] T. Oishi, K. Furuta and S. Kondo, "Workspace Recognition and Navigation of Autonomous Mobile Robot Using Self-Creating and Organizing Neural Network,” Transaction of the Society of Instrument and Dontrol Engineers, Vol. 33, No. 3, 1997, pp. 203-208.

[12] G. J. Choi and D. S. Ahn, "Map Building and Localiza- tion on Autonomous Mobile Robot Using Graph and Fuzzy Inference System,” IEEE International Joint Conference on Neural Networks, Budapest, 25-29 July 2004, pp. 2419-2424.

[13] Y. Zhuang, X. D. Xu and W. Wang, "Mobile Robot Geometric-Topological Map Building and Self-Localization," Control and Decision, Vol. 20, No. 7, 2005, pp. 815-818.

[14] A. Kawewong, Y. Honda, M. Tsuboyama and O. Hasegawa, "Reasoning on the Self-Organizing Incremental Associative Memory for Online Robot Path Planning," IEICE Transactions on Information and Systems, Vol. E93-D, No. 3, 2010, pp. 569-582.

[15] X. G. Ruan and X. T. Xing, "Application of Autonomous Mapping Algorithm on a Desktop Robot System,” Proceedings of the 5th International Conference on Natural Computation, Tianjin, 14-16 August 2009, pp. 448-453. doi:10.1109/ICNC.2009.156

[16] T. M. Martinetz, S. G.Berkovich and K. J. Schulten, "Neural-Gas Network for Vector Quantization and Its Application to Ime-Series Prediction,” IEEE Transactions on Neural Networks, Vol. 4, No. 4, 1996, pp. 558-569. doi:10.1109/72.238311

[17] B. Fritzke, "Growing Cell Structures-A Self-Organizing Network for Unsupervised and Supervised Learning," Neural Network, Vol. 7, No. 9, 1994, pp. 1411-1460. doi:10.1016/0893-6080(94)90091-4

[18] D. Choi and S. Park, "Self-Creating and Organizing Neural Networks," IEEE Transactions on Neural Networks, Vol. 5, No. 4, 1994, pp. 561-575. doi:10.1109/72.298226

[19] D. Alahakoon and S. K. Halgamuge, "Dynamic Self-Organizing Maps with Controlled Growth for Knowledge Discovery," IEEE Transactions on Neural Networks, Vol. 11, No.3, 2000, pp. 601-614. doi:10.1109/72.846732

[20] F. Shen and O. Hasegawa, "An Incremental Network for On-Line Unsupervised Classification and Topology Learning," Neural Networks, Vol. 19, 2006, pp. 90-106. doi:10.1016/j.neunet.2005.04.006 